\title{
Monitoring of Cognitive Processes in Older Persons
}

\author{
Rory Sobolewski, Richard B. Reilly \\ Trinity Centre for Bioengineering \\ Trinity College Dublin \\ Dublin, Ireland \\ e-mail: sobolewr@tcd.ie
}

\author{
Rory Sobolewski, Simon Finnigan, Paul Dockree, \\ Kate O’Sullivan, Ian H. Robertson, Richard B. \\ Reilly \\ Trinity College Institute of Neuroscience \\ Dublin, Ireland
}

\begin{abstract}
Evaluation of cognitive function is an important tool in clinical care. A novel method of cognitive monitoring is proposed, whereby subjects complete three sessions of the popular past-time Sudoku while EEG is recorded. Data epochs surrounding a correct solution are extracted and compared to a resting condition. Mean EEG amplitude in the alpha and theta bands are examined by means of the temporal spectral evolution method, and significant results include a desynchronization of alpha amplitude coinciding with an increase in theta amplitude prior to a correct solution in healthy adults. These results indicate that Sudoku may function as a light-touch, non-invasive and enjoyable means of monitoring for deteriorations in cognitive status.
\end{abstract}

Keywords- EEG; Aging; Temporal Spectral Evolution; Monitoring; Cognitive Decline

\section{INTRODUCTION}

At present, there are 30 million people worldwide who have been diagnosed with clinical dementia [1]. Dementia, while being a concern for all age groups, does primarily affect adults over the age of 65. As the world's population is aging due to improved diet, education and medication, the number of cases of dementia is set to increase to 100 million by the year 2050 [1] with a corresponding large strain on healthcare systems. As is often the case, early detection and prevention of diseases which lead to cognitive impairment (such as dementia) is the most effective method of treatment [2]. The problem with this approach however is that cognitive assessment is expensive and time consuming for the patient and the physician, and requires resources that only a minority has access to. There is therefore a pressing need to develop inexpensive, light-weight technologies that will provide feedback on a subject's cognitive status from an in-home environment on a regular basis.

In the study reported here, we propose a paradigm whereby EEG is employed to record electrophysiological data from the brain while the subject engages in a cognitively stimulating task. We introduce the puzzle Sudoku as that cognitive task, in the hope that the puzzle's popularity will offer an incentive to subjects to undertake it on a regular basis, thus providing continuous update of their cognitive state. The task is well suited to the monitoring of cognitive state, as it requires constant engagement on the part of the participant, as well as involving the processes of working memory and focused attention.

Our analysis focused on time locked EEG activity surrounding a correct solution in Sudoku, and its deviation from resting EEG. The alpha band $(8-12 \mathrm{~Hz})$ and the theta band $(4-8 \mathrm{~Hz})$ were taken as the frequency ranges to analyze, as these have been shown to be modulated directly by processes of attention [3] and memory [4], and are ultimately affected by cognitive decline [5], [6]. The aim of this study is to investigate if this paradigm can be used to monitor for significant changes in EEG in response to an ecological, real-life task, as oppose to classical tasks such as P300, VEP, n-back etc., which can be tedious and repetitive for the subject. If so, monitoring for cognitive decline may become more of a daily routine, and thus more cases of neurological impairment diagnosed in their earlier stages, leading to earlier intervention.

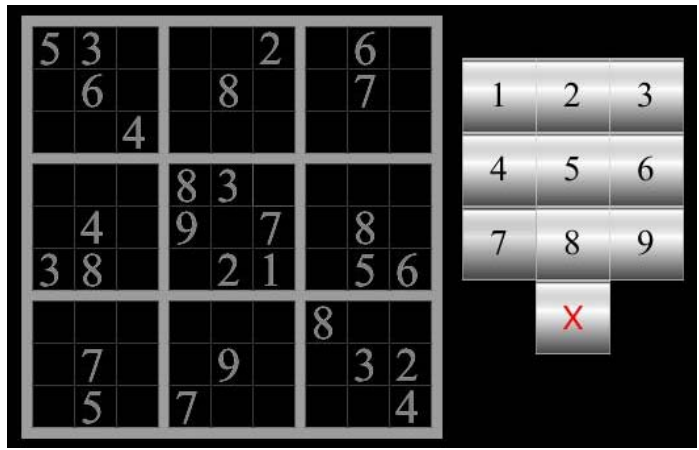

Fig. 1. Screenshot of the Sudoku GUI during a typical session

\section{METHODS}

\section{A. Subjects}

37 adults volunteered to participate in this study. 22 of the volunteers were aged between 19 and 38 (mean $=28.4$, S.D. $=$ 5.2), 12 of whom were male. The remaining 15 adults were aged between 61 and $79($ mean $=69.2$, S.D. $=5.1), 8$ of whom were male. The group of older adults was selected based on a previous battery of cognitive tests that provided a measure of cognitive status. Subjects with an MMSE (mini-mental state exam) score of 26 or greater were identified and recruited for the study [7]. All participants had normal or correct to normal vision. All participants gave their written consent prior to participation and the study was approved by the Trinity College Research Ethics Committee.

\section{B. Experimental Setup}

A graphical user interface (GUI) was designed specifically for the purposes of recording behavioral activity during Sudoku (Figure 1). Triggers were sent from the Sudoku PC to the data 

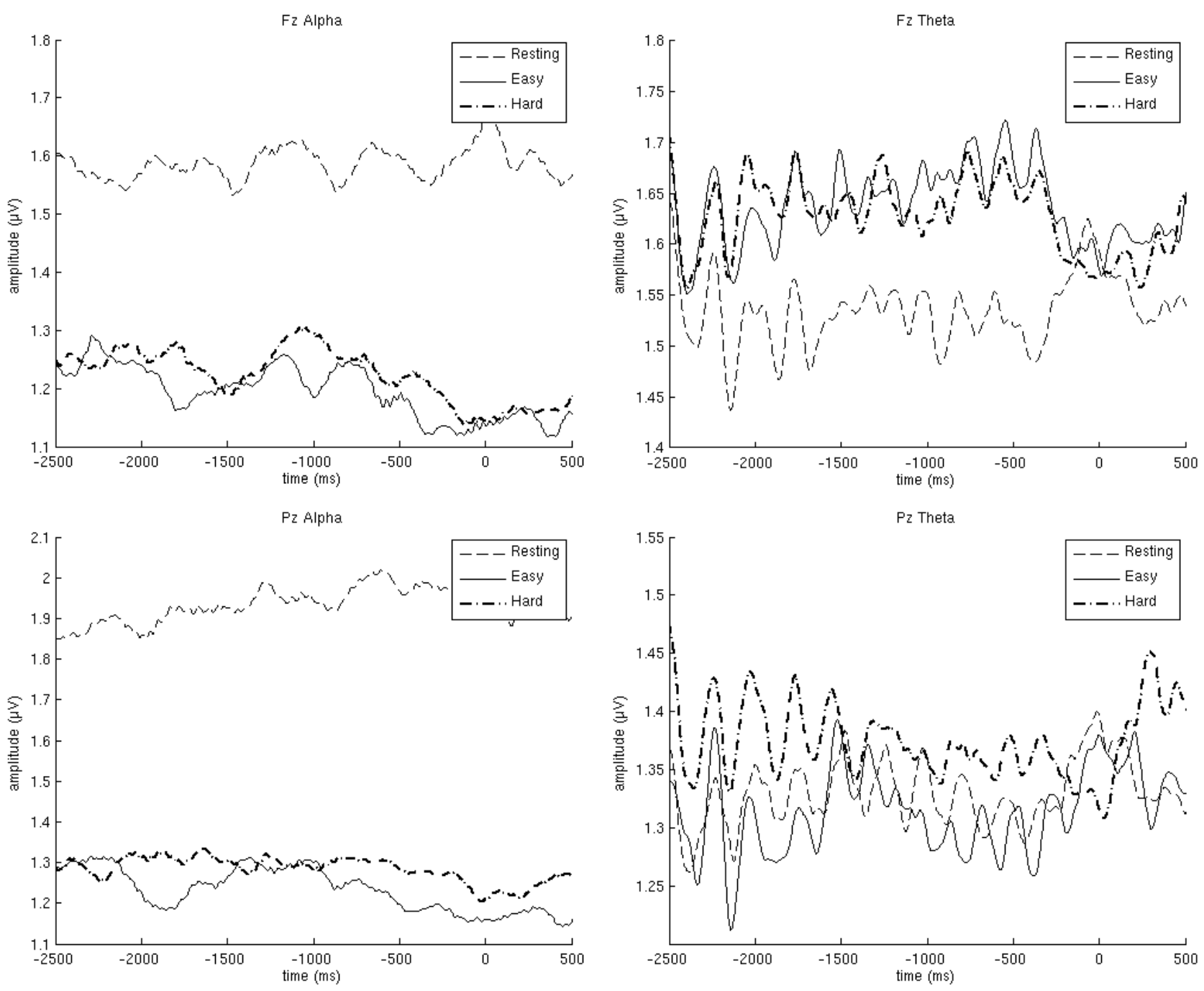

Fig. 2. Alpha (left) and theta (right) band TSEs at a frontal (top) and posterior (bottom) electrode location. Solution is arrived at at time $=0 \mathrm{~ms}$.

acquisition $\mathrm{PC}$ when the user began or stopped moving the mouse, as well as when any action on the GUI was implemented, such as a button press or completion of a session. All experimentation was carried out in a purpose built EEG facility in the Trinity College Institute of Neuroscience. Subjects were seated on a comfortable chair and positioned $1.5 \mathrm{~m}$ away from a CRT computer monitor. EEG data were recorded using the BioSemi ActiveTwo system [8]. 64 channels of EEG data were recorded with active scalp electrodes at a rate of $512 \mathrm{~Hz}$ per channel, and digitized with 24-bit resolution. Four external EOG electrodes were also included in order to record horizontal and vertical eye movement. Reference signals were recorded from both mastoids and tip of the nose.

\section{Tasks}

\section{1) Resting EEG}

Subjects were instructed to fixate on a crosshair in the centre of the screen for two minutes, and asked to keep eye blinks to a minimum while EEG was recorded.

\section{2) Sudoku Task}

All subjects undertook a training session of Sudoku in order to become familiar with the graphical user interface. Subjects then completed three sessions of Sudoku, the first being the least complicated requiring fewer answers and trivial solving strategies, the subsequent two becoming more difficult requiring more advanced solving strategies, based on the performance of the subject in the previous session.

\section{Data Analysis}

\section{1) EEG data analysis}

All processing of EEG data was carried out off line using the EEGLAB [9] toolbox for MATLAB [10]. The temporal spectral evolution (TSE) amplitude was computed prior to a correct solution for two levels of Sudoku, the most trivial session (Easy) and the most difficult session (Hard), and these compared to the resting EEG condition. The method for calculating the TSE as described in [11] is uncomplicated: EEG data were epoched based on a trigger sent by the Sudoku interface indicating a correct solution had been arrived at. As 
we are interested in the processes leading up to a solution, an epoch spanning from 3 seconds prior to the trigger up to 1 second after the trigger was examined, and was baseline corrected based on the first $0-100 \mathrm{~ms}$. The rejection threshold for epochs was $\pm 100 \mu \mathrm{V}$. Epochs were pass-band filtered to extract the frequency ranges of interest; in this case the alpha band from $8-12 \mathrm{~Hz}$ and the theta band from $4-8 \mathrm{~Hz}$. Epochs were then rectified, before being averaged together and finally smoothed by a $100 \mathrm{~ms}$ moving window. Alpha and Theta band TSEs from electrode sites $\mathrm{Fz}$ and $\mathrm{Pz}$ are displayed in Figure 2.

\section{2) Behavioral data}

The quantitative measure of performance in the Sudoku trials was taken as the average number of correct solutions minus the average number of incorrect solutions per minute, or

$$
s^{\prime}=\frac{(s-i)}{t},
$$

where $s$ and $i$ are the total number of correct and incorrect solutions respectively in a session of Sudoku, and $t$ is the time of that session in minutes. $s^{\prime}$ can then be thought of as the average solution rate of that session. In our analysis, we include only two levels of Task Difficulty, the solution rate taken from the most trivial (Easy) session and the solution rate from the most difficult (Hard) session that each subject successfully completed, in order to maximize the difference in effect size.

\section{3) Statistical analysis}

The mean TSE amplitude for each subject for each session of Sudoku was computed prior to a correct solution, and this was taken as the dependant variable for statistical analysis of the EEG data. A 3-way analysis of variance (ANOVA) was carried out for both alpha and theta TSEs, with a between subjects factor of Age and within subjects factors of Task Difficulty and Electrode, to investigate if age, difficulty or electrode placement had an effect on the mean TSE amplitude. The performance data was submitted to a 2-way ANOVA as the dependant variable, with Age as the between subjects factor and Task Difficulty as the within subjects factor. Post-hoc tests were carried out by adjusting $\alpha$ based on the conservative Bonferonni criteria. Finally, linear regression and correlations were performed to investigate directly if there was any relationship between TSE amplitude and performance.

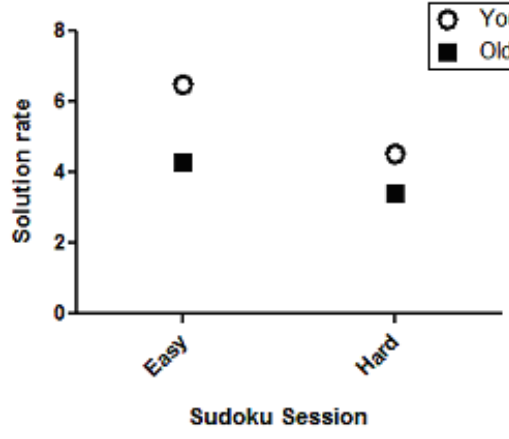

Fig. 3. Performance of younger and older adults for two levels of Sudoku

\section{RESULTS}

\section{A. Behavioral Data}

The 2-way ANOVA yielded a main effect of Task Difficulty, indicating faster solution rates in the Easy $\left(\mathrm{s}^{\prime}=5.3\right)$ compared to the Hard $\left(s^{\prime}=3.9\right)$ session $(p=0.001)$. A main effect of Age was also found with younger adults achieving on average 2 more solutions per minute than older adults $(\mathrm{p}<$ 0.001). No Age $\times$ Task Difficulty interaction was found however, which would suggest that performances in both age groups were affected equally in response to change in difficulty (Figure 3).

\section{B. EEG Data}

Analysis of variance was carried out on the mean TSE amplitudes computed from the alpha and theta frequency bands separately: For alpha TSE amplitude, a main effect of Task Difficulty was found indicating that alpha amplitude was reduced for the Sudoku Easy $(1.22 \mu \mathrm{V})$ and Hard $(1.27 \mu \mathrm{V})$ conditions compared to the Resting EEG $(1.66 \mu \mathrm{V})$ case $(p<$ 0.001 ). No effect of Age or Electrode was found. For theta TSE amplitude, a similar main effect was found for Task Difficulty, indicating an increase in theta amplitude prior to a solution in the Easy $(1.45 \mu \mathrm{V})$ and Hard $(1.49 \mu \mathrm{V})$ sessions compared to the Resting $(1.28 \mu \mathrm{V})$ condition $(p=0.01)$. A main effect of Age was found, suggesting theta amplitude was greater in younger $(1.51 \mu \mathrm{V})$ than older $(1.3 \mu \mathrm{V})$ adults $(p=0.001)$ over all conditions. A main effect of Electrode was also found, indicating larger theta amplitude at frontal $(1.52 \mu \mathrm{V})$ electrode sites compared to posterior $(1.29 \mu \mathrm{V})$ sites $(p<0.001)$. These findings are further explained by the significant Age $\times$ Electrode interaction $(p=0.03)$ which highlights an increase in theta amplitude in younger adults specifically at frontal sites compared to older adults, while amplitude at posterior sites is not significantly different between groups. Finally, an Age $\times$ Difficulty $\times$ Electrode interaction was significant $(p=0.05)$ which indicated that as the difficulty of the task increased from resting to Easy or to Hard, theta amplitude at frontal locations increased in the younger adult (Easy: $1.84 \mu \mathrm{V}$, Hard: $1.83 \mu \mathrm{V}$ ) group, but not to the same extent for the older adult group (Easy: $1.36 \mu \mathrm{V}$, Hard: $1.37 \mu \mathrm{V}$ ), and again remained nonsignificantly different at posterior locations. The results for the 3-way ANOVA with theta TSE amplitude as dependant variable are recorded in Table 1.

TABLE I

TESTS OF BETWEEN-SUBJECTS EFFECTS

\begin{tabular}{lcl}
\hline \hline \multicolumn{1}{c}{ Source } & F & Sig. \\
\hline Age & 11.53 & $0.001^{* *}$ \\
Task Difficulty & 4.36 & $0.014^{*}$ \\
Electrode & 14.18 & $<0.001 * *$ \\
Age $\times$ Task Difficulty & 0.73 & 0.48 \\
Age $\times$ Electrode & 4.45 & $0.03 *$ \\
Task Difficulty $\times$ Electrode & 0.48 & 0.61 \\
Age $\times$ Task Difficulty $\times$ Electrode & 2.65 & 0.05 \\
\hline \hline
\end{tabular}

Between-subjects effects with mean theta TSE amplitude as dependant variable.

${ }^{*} \mathrm{p}<.05 * * \mathrm{p}<.01$ 


\section{Behavioral vs. EEG Data}

Regression and correlation were carried out to determine if any relationship existed between mean alpha or theta TSE amplitude and performance in the Sudoku task, but no significant correlations were found at any level of difficulty for either age group.

\section{DISCUSSION}

This study investigated the possibility of employing the puzzle Sudoku as a means of monitoring for changes in an individual's cognitive state, by analyzing elicited EEG data prior to a correct solution. By comparing the loading effect of a Sudoku puzzle compared to a resting condition on a group of adult volunteers, our results indicated that the Sudoku task did elicit responses indicative of the processes being examined. In compliance with the literature, alpha amplitude significantly decreased in the epoch examined prior to a solution, although from visual inspection of the TSEs at frontal and posterior sites, this decreases does not appear to be locked in time to the solution trigger. Instead, alpha amplitude appears to remain consistently lower for the task in general, indicating the subjects' attention was sustained and fixed throughout the entire session, not just focused at specific points in time. As alpha activity is well known to reflect attentional processes and to desynchronize with attention [12], the Sudoku task may function as a means of monitoring levels of engagement during a single duration, or to highlight any long term inconsistencies in the user's ability to maintain an attentive state over the course of many trials.

Theta amplitude conversely was found to increase prior to a correct solution being arrived at, although this increase in amplitude was confined to frontal as oppose to posterior electrode sites. This is consistent with studies which noted an increase in theta amplitude during the recall of information from memory [13], [14]. As can be seen in the figure labeled 'Fz theta' in Figure 2, this increase in theta amplitude does seem to be time locked to a correct solution, which suggests that the information required to obtain a solution was being retrieved from memory and acted upon before a solution was entered. Again this validates the Sudoku paradigm as a means of eliciting relevant neurological data, as theta activity is known to be altered with cognitive decline [15] [16] including the cognitive decline associated with normal aging [17]. Furthermore, a decrease in performance was associated with the group of older adults (Figure 3) which suggests that the underlying neural mechanisms that gave rise to the recorded electrophysiological differences between the two groups namely a reduction in alpha desynchronisation and reduced theta amplitude in the older adult group- may also be detrimental to cognitive function, in concurrence with the aforementioned literature.

Future research will involve a longitudinal study with the aim of monitoring changes in the elicited signals over time, and quantifying their usefulness as a means of monitoring for the early detection of cognitive decline.

\section{CONCLUSION}

A novel method of cognitive monitoring is proposed, that elicits consistent electrophysiological signals in the human brain. The ability to use cognitively stimulating, ecological tasks as a window into cognitive function opens the possibility to better understand the neurodegenerative process. Having such a task and analysis framework also allows remote and long term monitoring of cognitive status, offsetting current labour intensive methods. With an increasingly aging population, long term monitoring in this manner will hopefully lead to new interventions for neurodegenerative diseases.

\section{REFERENCES}

[1] Alzheimer's Disease International, http://www.alz.co.uk

[2] C. D. Smith, H. Chebrolu, D.R. Wekstein, F.A. Schmitt, G.A. Jicha et al., "Brain structural alterations before mild cognitive impairment," Neurology, vol. 68, Apr. 2007, pp. $1268-1273$

[3] W. Klimesch, M. Doppelmayr, H. Russegger, T. Pachinger and J. Schwaiger, "Induced alpha band power changes in the human EEG and attention," Neuroscience Letters, vol. 244, Mar. 1998, pp. 73 - 76

[4] W. Klimesch, M. Doppelmayr, H. Russeger and T. Pachinger, "Theta band power in the human scalp EEG and the encoding of new information," Neuroreport, vol. 7, May 1996, pp. 1235 - 1240

[5] C. Clark, M.D. Veltmeyer, R.J. Hamilton, E. Simms, R. Paul et al., "Spontaneous alpha peak frequency predicts working memory performance across the age span," Inernational. Journal of Psychophysiology, vol. 53, Jun. 2004, pp. 1 -9

[6] R.A.P. Roche, P.M. Dockree, H. Garavan, J.J. Foxe, I.H. Robertson et al., "EEG alpha power changes reflect response inhibition deficits after traumatic brain injury(TBI) in humans," Neuroscience Letters, vol. 362, May 2004, pp. $1-5$

[7] M.F. Folstein, S.E. Folstein and P.R. McHugh, "Mini-Mental State: A practical method for grading the cognitive state of patients for the clinician," Journal of Psychiatric Research, vol. 12, Nov. 1975, pp. 189 $-198$

[8] http://www.biosemi.com/products.htm

[9] A. Delorme and S. Makeig, "EEGLAB: an open source toolbox for analysis of single-trial EEG dynamics including independent component analysis," Journal of Neuroscience Methods, vol. 134, Mar. 2004, pp. 9 $-21$

[10] http://www.mathworks.com-/products/matlab/

[11] S.P. Kelly, M. Gomez-Ramirez, J.L. Montesi and J.J. Foxe, "L-theanine and caffeine in combination affect human cognition as evidenced by oscillatory alpha-band activity and attention task performance," Journal of Nutrition, vol. 138, Aug. 2008, pp. 1572S - 1577S

[12] W. Klimesch, "EEG alpha and theta oscillations reflect cognitive and memory performance: a review and analysis," Brain Research Reviews, vol. 29, Apr. 1999, pp. $169-195$

[13] P. Sauseng, W. Klimesch, M. Doppelmayr, S. Hanslmayr, M. Schabus et al., "Theta coupling in the human electroencephalogram during a working memory task," Neuroscience Letters, vol. 354, Jan. 2004, pp. $123-126$

[14] A.P. Burgess and J.H. Gruzelier, "Short duration synchronization of human theta rhythm during recognition memory," Neuroreport, vol. 8, Mar. 1997, pp. 1039 - 1042

[15] J. Duff, "The usefulness of quantitative EEG (QEEG) and neurotherapy in the assessment and treatment of post-concussion syndrome," Clinical EEG and Neuroscience, vol. 35, Oct. 2004, pp. 198 - 209

[16] J. Johnstone and R.W. Thatcher, "Quantitative EEG analysis and rehabilitation issues in mild traumatic brain injury," Journal of Insurance Medicine, vol. 23, Dec. 1991, pp. 228 - 232

[17] T.D.R. Cummins and S. Finnigan, "Theta power is reduced in healthy cognitive aging," International Journal of Psychophysiology, vol. 66, Oct. 2007 , pp. $10-17$ 